\section{Public Health Genomics}

Public Health Genomics 2013;16:118-126

DOI: $10.1159 / 000349924$
Received: December 12, 2012

Accepted after revision: February 19, 2013

Published online: April 12, 2013

\title{
Potential Bias in the Bank: What Distinguishes Refusers, Nonresponders and Participants in a Clinic-Based Biobank?
}

\author{
J.L. Ridgeway ${ }^{a} \quad$ L.C. Han ${ }^{b} \quad$ J.E. Olson ${ }^{c} \quad$ K.A. Lackore ${ }^{a} \quad$ B.A. Koenig ${ }^{\text {e }} \quad$ T.J. Beebe ${ }^{a}$ \\ J.Y. Ziegenfuss ${ }^{d}$ \\ ${ }^{a}$ Division of Health Care Policy and Research, ${ }^{\mathrm{b}}$ Biomedical Ethics Research Unit, and ${ }^{\mathrm{c} D i v i s i o n}$ of Epidemiology, \\ Mayo Clinic, Rochester, Minn., 'D Data Collection Center, HealthPartners Institute for Education and Research, \\ Minneapolis, Minn., and 'Institute for Health and Aging, University of California, San Francisco, Calif., USA
}

\section{Key Words}

Biobank $\cdot$ Informed consent $\cdot$ Nonresponse $\cdot$ Research participation

\begin{abstract}
Background: Biobanks are an important resource for genetic and epidemiologic research, but bias may be introduced if those who accept the recruitment invitation differ systematically from those who do not in terms of attributes important to health-related investigations. To understand potential bias in a clinic-based biobank of biological samples, including genetic data linked to electronic health record information, we compared patient characteristics and self-reported information among participants, nonresponders and refusers. We also compared reasons for nonparticipation between refusers and nonresponders to elucidate potential pathways to reduce nonparticipation and any uncovered bias. Methods: We mailed recruitment packets to 1,600 adult patients with upcoming appointments at Mayo Clinic (Rochester, Minn., USA) and recorded their participation status. Administrative data were used to compare characteristics across groups. We used phone interviews with 26 nonresponders and 26 refusers to collect self-reported information, including reasons for nonparticipation. Participants were asked to complete a mailed questionnaire. Results: We achieved $26.2 \%$ participation $(n=419)$ with $12.1 \%$ refusing
\end{abstract}

$(\mathrm{n}=193)$ and $61.8 \%$ nonresponse $(\mathrm{n}=988)$. In multivariate analyses, sex, age, region of residence, and race/ethnicity were significantly associated with participation. The groups differed in information-seeking behaviors and research experience. Refusers more often cited privacy concerns, while nonresponders more often identified time constraints as the reason for nonparticipation. Conclusion: For genomic medicine to advance, large, representative biobanks are required. Significant associations between patient characteristics and nonresponse, as well as systematic differences between refusers and nonresponders, could introduce bias. Oversampling or recruitment changes, including heightened attention to privacy protection and participation burden, may be necessary to increase participation among less-represented groups.

Copyright $\odot 2013$ S. Karger AG, Basel

\section{Introduction}

Biobanks are an increasingly important method of collecting health-related information for research. Some of these biobanks include access not only to a biorepository of genetic materials, but also access to linked medical record data and self-reported health information gathered through questionnaires. Biobanks aim to be large enough to facilitate research on a number of diseases, some of

\section{KARGER}

E-Mail karger@karger.com

www.karger.com/phg
(C) 2013 S. Karger AG, Basel

1662-4246/13/0163-0118\$38.00/0
Jennifer L. Ridgeway

Mayo Clinic Rochester, Health Sciences Research, Rosa Parks Pavilion Building 3-26 200 First Street SW

Rochester, MN 55905 (USA)

E-Mail ridgeway.jennifer@ mayo.edu 
which may have higher prevalence in certain populations. Failure to include a large and diverse population limits researchers' ability to use the biobank for research that requires large numbers of samples from specific subsets of the population. Furthermore, to the extent that characteristics of less-represented persons are associated with disease-related genetic variants, a less-diverse collection may hamper research in genetic links to disease or treatment.

Research relying on biobank data linked to health information, especially self-reported questionnaire data, raises additional questions about representativeness. Like most forms of health research, less than complete participation raises concerns over selection bias and representation, whereby those who accept the invitation to supply their health information to a biobank may differ systematically from those who do not in terms of attributes important to health-related investigations. Bias in questionnaire data, which may help identify environmental or social risk factors related to health outcomes, further limits the potential of biobanks to serve as a resource to explore the interaction of genetics, patient characteristics, risk factors, and outcomes.

In order to understand issues related to potential bias in biobanks that would limit representativeness, researchers must understand how patient characteristics are associated with participation and nonparticipation. Prior research suggests that biobank participation may be associated with characteristics including age and race, but there is limited research on characteristics associated with refusal to participate compared to nonresponse to an invitation to participate in a clinic-based biobank [1-3].

To increase the numbers and diversity of samples in a biobank, researchers must further study participant motivations and barriers to participation. People who choose to participate in biobanks have been found to do so for a variety of reasons, including potential benefits to self, family or future generations, and a desire to help advance science [4-7]. Reasons for nonparticipation identified by previous studies include lack of time, inconvenience, need for a blood draw, privacy and confidentiality concerns, mistrust of institutions, and discomfort about genetic research $[2,5,6,8-11]$. However, much of what is known about biobank participation is limited to people who had contact with a biobank staff, i.e. participants and active refusers, or is limited to participants versus nonparticipants, thereby failing to capture differences between refusers and nonresponders. The existing literature is also somewhat limited to results based on individuals' views of whether they would participate in the future, rather than concurrent biobank recruitment, or to individuals being recruited to disease-specific biobanks that may be of specific interest to a narrower pool of potential participants. This study was designed to overcome these limitations by comparing characteristics of participants, nonresponders and active refusers - as well as the major reasons for not participating among the latter 2 groups - during actual recruitment to a large clinic-based biobank located in Southeast Minnesota.

\section{Methods}

\section{Setting and Participant Recruitment}

The Mayo Clinic Biobank is characterized as a collection of biological samples as well as patient-reported health information from a mailed survey and information in the electronic health record (http://mayoresearch.mayo.edu/mayo/research/biobank/). The Mayo Clinic Biobank is not disease-specific. It includes samples and health information from patients and healthy volunteers age 18 and older regardless of health history. At the time of this study, the biobank included samples and information for more than 20,000 patients $(58 \%$ female and $95 \%$ white, with a median age of 62 years). When patients consent to biobank participation, they agree to allow researchers to access these data for future studies and to share data though federal de-identified biorepositories.

The biobank recruits participants by mail and through recruitment desks in 2 locations of Mayo Clinic in Rochester, Minn., USA. The mail recruitment sample is randomly drawn from patient appointment calendars, with recruitment targeted at primary care clinic appointments. Selected individuals are sent a mailed invitation to participate in the biobank. We limited this study to individuals recruited by mail over a 1-month period in August 2011. During this time period, 1,600 biobank recruitment packets were mailed. The packets included general information about the biobank, a consent form, a form to select an incentive worth USD 20 , a health questionnaire (containing questions about health behaviors, environmental exposures and family health history), and instructions for providing a blood sample. Half of the potential participants over this time period were randomized to packets including the original consent form, and half to packets including a simplified consent form designed specifically for this study. A study comparing participant comprehension of informed consent information by form type is reported elsewhere [12].

The original consent form, in use since biobank recruitment began in 2009, was developed with input from the Mayo Clinic Biobank community advisory board (CAB). At that time, the CAB advocated for concise documents in the informed consent process, but regulatory and institutional requirements eventually led to a longer and more detail-heavy consent form than the one proposed. The simplified form developed for this study built on the work of those involved in developing the original form as well as a review of the literature on plain language and readability in research documents, including recent work on developing simplified consent forms [13-20]. A draft of our simplified form was reviewed by members of the $\mathrm{CAB}$ and an external ethics consultant. Relative to the original form, the simplified form was shorter (6 vs. 11 pages), used language at a lower reading-grade level (7.9 vs. 9.8), included 


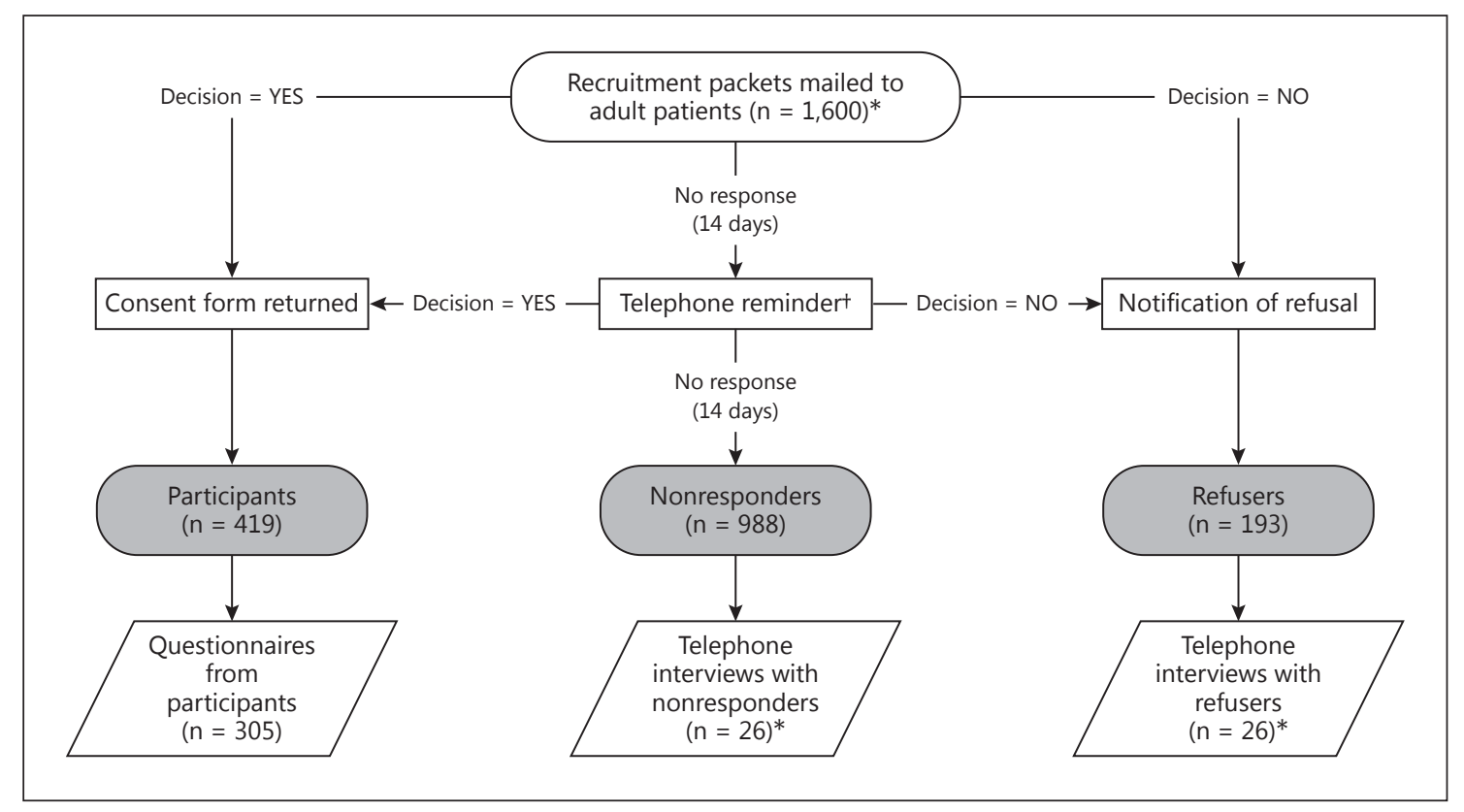

Fig. 1. Recruitment process, participation decisions and data collection. The study process started with recruitment of participants to the biobank. Based on their response to the invitation, individuals were classified as participants, nonresponders or refusers. Participants were asked to complete a mailed questionnaire, while re- fusers and nonresponders were recruited for a telephone interview. * Stratified by original and simplified consent form type. ${ }^{\dagger}$ The study protocol was for patients to receive a phone call reminder after 14 days. Due to staffing issues, not all patients received a call. illustrations (4 pictures vs. none), and eliminated passive-voice sentences. Length was shortened by reducing redundancies without sacrificing important content. All ethical and legal requirements were still met in the simplified version of the form. The Mayo Clinic Institutional Review Board approved this study. All patients in the recruitment pool received a cover letter describing this study that provided them the opportunity to opt out of the biobank, this study or both.

\section{Study Design}

The recruitment process and data collection steps are shown in figure 1. All patients in the recruitment pool were coded to one of 3 types of participation status: participant, refuser and nonresponder. Patients were considered participants for this study when they returned a signed biobank consent form to the study staff. We did not track participants to see if they completed the eventual blood draw or the health questionnaire necessary for biobank participation. The biobank protocol called for patients to receive a follow-up phone call after 14 days if there was no response to the recruitment packet. If there was no response after an additional 14 days, patients were considered to be nonresponders. Patients were classified as refusers if they checked a box on the recruitment letter indicating their desire to opt out of the biobank and returned it to the biobank staff. Patients could also opt out of this additional study by checking another box on the recruitment letter. Recruitment status was verified for all patients after the study period ended on December 15, 2011.

The study staff tracked the number and time of contacts and attempted contacts during recruitment, as well as when responses were received, and this information was stored in the study database. Patient characteristics, including age and sex, were identified from registration records for all patients in the study.

Patients who consented to biobank participation were sent a follow-up questionnaire specific to this study within 14 days of consent to gather information about personal characteristics and views on research. Also assessed at this time was comprehension of information included in the consent forms. Discussion of the results on informed consent comprehension is presented elsewhere [12].

Refusers and nonresponders to the biobank who did not opt out of this study were contacted by trained telephone interviewers from the Mayo Clinic Survey Research Center for a semistructured interview about their reasons for nonparticipation and to gather information about personal characteristics and views about research. Respondents were assured that the interviewers were not trying to change their mind, but rather were simply attempting to get information about their reasons for not participating. Interviewers used prompts to elicit further detail on reasons for nonparticipation if respondents said they were too busy or did not have time to participate. Interviewers contacted refusers and nonresponders until they completed 26 interviews in each group (with each group stratified by consent form type: original or simplified version). Interviews were audio recorded for those patients who provided verbal consent (fig. 1.)

\section{Analysis Methods}

Quantitative Analysis. We began with comparison of basic patient characteristics and response rates across the 3 groups. We 
Table 1. Multivariate-adjusted ORs for participation, nonresponse, and refusal to participate in the biobank

\begin{tabular}{|c|c|c|c|c|c|c|c|c|}
\hline \multirow[t]{2}{*}{ Characteristic } & \multicolumn{2}{|c|}{$\begin{array}{l}\text { Model 1: participant vs. } \\
\text { nonparticipation }^{\mathrm{a}}(\mathrm{n}=1,600)\end{array}$} & \multicolumn{2}{|c|}{$\begin{array}{l}\text { Model 2: nonresponder } \\
\text { vs. participant }(n=1,407)\end{array}$} & \multicolumn{2}{|c|}{$\begin{array}{l}\text { Model 3: refuser vs. partici- } \\
\text { pant }(n=612)\end{array}$} & \multicolumn{2}{|c|}{$\begin{array}{l}\text { Model 4: refuser vs. nonre- } \\
\text { sponder }(n=1,181)\end{array}$} \\
\hline & adj. OR & $\mathrm{p}$ value & adj. OR & $\mathrm{p}$ value & adj. OR & $\mathrm{p}$ value & adj. OR & $\mathrm{p}$ value \\
\hline Simplified consent form & 0.67 & $<0.001$ & 1.53 & $<0.001$ & 1.31 & 0.15 & 0.85 & 0.32 \\
\hline Female & 1.38 & $<0.01$ & 0.67 & $<0.01$ & 0.97 & 0.87 & 1.49 & 0.02 \\
\hline \multicolumn{9}{|l|}{ Age category, years } \\
\hline $21-34$ & 1 [ref.] & & 1 [ref.] & & 1 [ref.] & & 1 [ref.] & \\
\hline $35-44$ & 1.38 & 0.21 & 0.69 & 0.15 & 1.10 & 0.82 & 1.61 & 0.21 \\
\hline $45-54$ & 1.92 & $<0.01$ & 0.47 & $<0.001$ & 1.07 & 0.85 & 2.24 & 0.02 \\
\hline $55-64$ & 2.14 & $<0.001$ & 0.38 & $<0.0001$ & 1.76 & 0.13 & 4.54 & $<0.0001$ \\
\hline $65-74$ & 2.77 & $<0.0001$ & 0.26 & $<0.0001$ & 2.00 & 0.06 & 8.18 & $<0.0001$ \\
\hline $75+$ & 2.49 & $<0.001$ & 0.27 & $<0.0001$ & 2.52 & 0.02 & 10.13 & $<0.0001$ \\
\hline \multicolumn{9}{|l|}{ Area of residence } \\
\hline Olmsted County & 1 [ref.] & & 1 [ref.] & & 1 [ref.] & & 1 [ref.] & \\
\hline Southeast $\mathrm{MN}^{\mathrm{b}}$ & 0.80 & 0.24 & 1.26 & 0.25 & 1.49 & 0.13 & 1.22 & 0.39 \\
\hline Other $\mathrm{MN}^{\mathrm{c}}$ & 1.49 & 0.02 & 0.74 & 0.11 & 0.48 & $<0.01$ & 0.59 & 0.04 \\
\hline IA, WI, ND, SD & 1.94 & $<0.001$ & 0.61 & 0.02 & 0.30 & $<0.001$ & 0.55 & 0.06 \\
\hline Other US & 1.45 & 0.03 & 0.81 & 0.24 & 0.43 & $<0.01$ & 0.51 & $<0.01$ \\
\hline Non-Hispanic white & 1.60 & 0.01 & 0.64 & 0.02 & 0.61 & 0.07 & 0.93 & 0.78 \\
\hline
\end{tabular}

adj. $\mathrm{OR}=$ Adjusted odds ratio; ref. $=$ reference.

${ }^{a}$ Nonparticipation includes nonresponders and refusers. ${ }^{\mathrm{b}}$ Southeast $\mathrm{MN}=$ eleven counties surrounding and including Olmsted County. ${ }^{\mathrm{c}}$ Other $\mathrm{MN}=$ all other counties in MN except those in Southeast MN.

compared responses on close-ended questions from refuser and nonresponder phone interviews as well as responses to questions that appeared in both the interview scripts and the participant questionnaire. Comparisons were made using the t test, $\chi^{2}$ test, or Fisher's exact test where appropriate. We used multivariable linear regression to determine whether consent form type, sex, age category, area of residence (based on the patient's home address), and race/ethnicity were independently associated with recruitment outcomes. A variable indicating whether the patient received a follow-up reminder phone call was included in exploratory analyses. All statistical analyses were performed using SAS version 9.2 (SAS Institute Inc., Cary, N.C., USA). A significance level of 0.05 was used for all statistical analyses.

Qualitative Analysis. The audio files of refuser and nonresponder interviews were imported into NVivo version 9 qualitative data analysis software (QSR International, Doncaster, Vic., Australia). A trained qualitative analyst listened to all audio files and transcribed verbatim the responses to open-ended questions directly in NVivo. Methods of content analysis were used to interpret the data. The analyst started with familiarization of the data and identification of a priori and emergent categories and then coded text to each of the categories. The coded text was analyzed within group (refusers and nonresponders). All interview responses fit into at least one of the identified categories. Analytic memos describing interpretation of the results for the 2 groups were shared with the study team for review. Coded text was grouped into themes and translated into quantitative data in order to compare frequencies of reasons between refusers and nonresponders. Although we did not test for significant differences between these groups, these data show the intensity of themes within each group [21].

Biobank Nonresponse, Refusal and Participation

\section{Results}

\section{Quantitative Results}

Patient Characteristics and Response Rates

Our data file included the participation decision and demographic characteristics for all of the patients in the recruitment pool $(n=1,600)$. Over the study period, the biobank achieved a $26.2 \%$ participation rate $(n=419)$ with an additional $12.1 \%$ actively refusing participation $(\mathrm{n}=193)$ and $61.8 \%$ not responding $(\mathrm{n}=988)$. Table 1 shows the results of 4 multivariate logistic regression models. In model 1, female sex, age categories 45 and older, residence outside the 11-county region where Mayo Clinic is located, and non-Hispanic white race/ethnicity were positively associated with participation relative to nonparticipation. The simplified consent form was associated with lower odds of participation. In model 2, nonresponse relative to participation, we found that the simplified form was associated with increased odds of nonresponse, while female sex, age categories 45 and older, residence outside of Minnesota, and non-Hispanic white identification were associated with lower odds of nonresponse. In the model of refusal versus participation, model 3, age 75-plus was associated with increased odds of refusal, while residence outside the region was associated 
Fig. 2. Biobank knowledge and research participation by group. Refusers, nonresponders and participants were asked whether they had heard of the Mayo Clinic Biobank before receiving the recruitment packet in the mail. They were also presented with a list of resources, such as the biobank staff and family member, and asked whether they had consulted any of these resources or any other resource in making their participation decision. Individuals who consulted any resource are included as having looked for information in the second set of columns. Individuals were asked to report how much of the consent form they read in 5 categories ranging from 'all of it' to 'none of it'. The final set of columns displays those who reported that they read all or most of the document. $p$ values were calculated using Fisher's exact test.

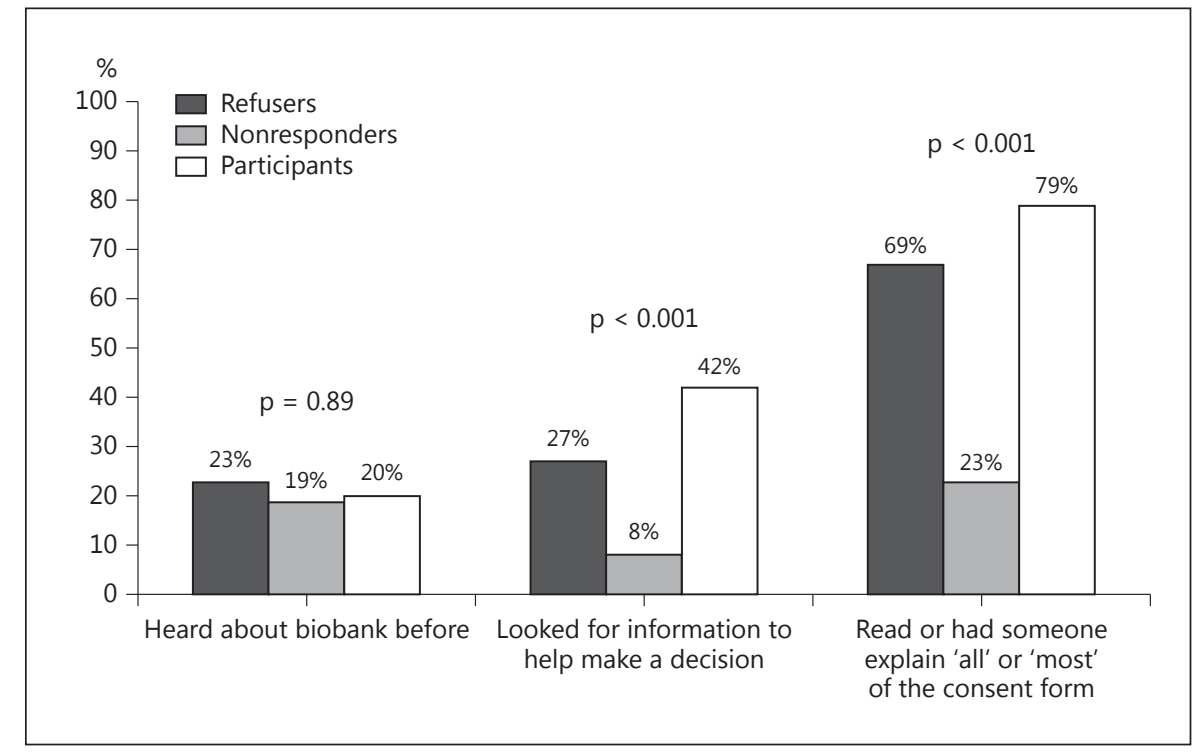

with lower odds of refusal. In model 4, refusal versus nonresponse, female sex and older age categories were associated with increased odds of refusal, while living in other areas of Minnesota or in states outside the Midwest was associated with lower odds of refusal (table 1).

\section{Biobank Knowledge}

We received 305 study questionnaires from the 419 participants (72.8\% response) and completed telephone interviews with 26 nonresponders and 26 refusers. Among refusers and nonresponders contacted for a phone interview, there were no significant differences on sex, age, area of residence, or race/ethnicity between those who agreed to phone follow-up and those who did not. Among participants, there were significant differences on age and race/ethnicity between those who returned the study questionnaire and those who did not, with higher shares of older participants and non-Hispanic white participants returning the questionnaire.

As shown in figure 2, participants, refusers and nonresponders had similar knowledge but differing information seeking behavior. Only $20 \%$ of participants had prior knowledge of the Mayo Clinic Biobank, compared with $23 \%$ of refusers and $19 \%$ of nonresponders (differences not significant). There were significant differences between groups when people were asked if they looked for information to help them make a decision about participating in the biobank ( $42 \%$ of participants, $26.9 \%$ of refusers and $7.7 \%$ of nonresponders, $\mathrm{p}<0.001$ ). Respondents were specifically asked if they consulted the biobank staff (in person or by phone), a family member, a friend or coworker, a health care provider, the internet, or some other source. People who looked for information most often cited family members as their source, followed by the biobank staff (in person or by phone). Fewer people consulted a friend or coworker, a health care provider, or the internet.

There were also significant differences in the share of people who read all or most of the consent form $(79 \%$ of participants, $69 \%$ of refusers and $23 \%$ of nonresponders, $p$ $<0.001)$. Among participants, more than three-fourths of patients read all or most of the form regardless of whether they received the original or the simplified version (77 vs. $74 \%$, respectively; $\mathrm{p}=0.52$ ). The proportion who read all or most of the form was higher for patients who received the simplified version than the original version in the refuser and nonresponder groups ( 84 vs. $53 \%, p=0.20$, and 30 vs. $15 \%, \mathrm{p}=0.64$, respectively).

\section{Views on Research}

As shown in figure 3, refusers were much more likely than nonresponders to have participated in medical research in the past ( 65 vs. $27 \%, \mathrm{p}=0.005$ ), but they were equally likely to claim that they would participate in the future. Findings were also similar on questions about confidentiality of medical information and privacy of genetic information, with about half of people in each group responding that they were concerned or very concerned about these issues (fig. 3). 
Table 2. Overview of reasons for nonparticipation in the Mayo Clinic Biobank, qualitative interview questions with refusers and nonresponders

\begin{tabular}{lll}
\hline Theme & Subtheme & Illustrative quote \\
\hline Too busy to participate & $\begin{array}{l}\text { Not enough time to } \\
\text { complete the study } \\
\text { materials }\end{array}$ & $\begin{array}{l}\text { 'I just looked at it, and it was so thick, it was several pages, and I thought } \\
\text { I just don't have time for that now.' [Male, age 53 (nonresponder)] }\end{array}$ \\
& $\begin{array}{l}\text { 'Well, I started to fill out the paperwork, I wanted to, but it was just way } \\
\text { too much to fill out and I was really busy.' [Female, age 70 (nonre- } \\
\text { sponder)] }\end{array}$
\end{tabular}

Not a priority given life demands
'I had 11 different things going on at once. It wasn't at the top of my list.' [Male, age 51 (nonresponder)]

'Just busy. Right now my life is planning a wedding and moving ... Just chose not to do it. I had other things that were a priority for me.' [Female, age 27 (nonresponder)]

Concerns about confidentiality of medical information or privacy of genetic information
Concerned about sharing information with researchers outside the Mayo Clinic
'I would be down there in 5 minutes if it was just strictly for Mayo. But this day and age, yes, they tell me you're a bar code, but I'm a firm believer that somewhere down the line that bar code is going to turn into a name.' [Male, age 71 (refuser)]
'I have participated in research at Mayo before, but this is a privacy issue. I don't think I want Mayo Clinic giving my information, especially my genetic information, to someone else.' [Male, age 70 (refuser)]

Concerns about electronic data storage

'Things are getting so, with the internet, you can find out a lot of stuff really easy. I guess personally I don't know anyone who has really been affected by that but you hear stories ... 5 years ago I would not have been concerned at all. I'm probably more concerned now.' [Female, age 55 (refuser)]

Concerns about information getting into the wrong hands
'When I was reading the consent, there was a part in there that said, I can't quite remember, that they wouldn't be able to protect or confirm that my blood sample would possibly be in the hands of future people who make decisions with insurance and life insurance and that kind of stuff.' [Female, age 51 (refuser)]

\section{Qualitative Results}

Interviews with refusers and nonresponders provided greater detail on reasons for nonparticipation. Table 2 lists the main themes from interviews: being too busy to participate and concerns about confidentiality. There were notable differences in the prevalence of these themes between refusers and nonresponders, as shown in figure 4. A majority of nonresponders $(n=19,73 \%)$ said they were too busy or didn't have time to complete one of the biobank participation requirements including reading the materials $(n=5)$ or completing the survey $(n=4)$. Most interviewees gave general feedback about being 'too busy', in many instances noting feeling overwhelmed by things going on in their life and biobank participation being a low priority for them.

In contrast, only 3 refusers noted being too busy as a reason for nonparticipation (12\%). One woman who stated a time constraint specified that she would have partici- pated if the survey was online; she indicated that the materials stated a tighter turnaround time than she was able to meet. Another said she might have participated if remuneration made it worth her while. Unlike nonresponders, this group did not state that they were 'just too busy' or indicate that they were overwhelmed by other things going on in their lives, although 3 people indicated poor health as being the reason for not participating. Instead patients cited specific reasons for refusing, such as decisions not to travel to Mayo Clinic for their care (biobank participants usually coordinate the blood draw with a scheduled clinic appointment time) or the inconvenience of traveling back and forth to Mayo Clinic (with 2 people noting that they believed there was a chance that they would need to travel to Mayo Clinic again for something related to the biobank study), or negative feelings about Mayo Clinic, going to the doctor, or giving blood. One noted a concern that Mayo Clinic could 'end up making a 
Fig. 3. Views on research, refusers and nonresponders. Refusers and nonrefusers were asked if they had ever participated in medical research in the past (yes/no) as well as being asked a question about whether they would participate in the future on a 4-point scale from 'very likely' to 'very unlikely'. In separate questions, respondents were asked how concerned they were about the confidentiality of their medical information and the privacy of their genetic information using a 4-point scale from 'very concerned' to 'not at all concerned'. p values based on $\chi^{2}$ test.

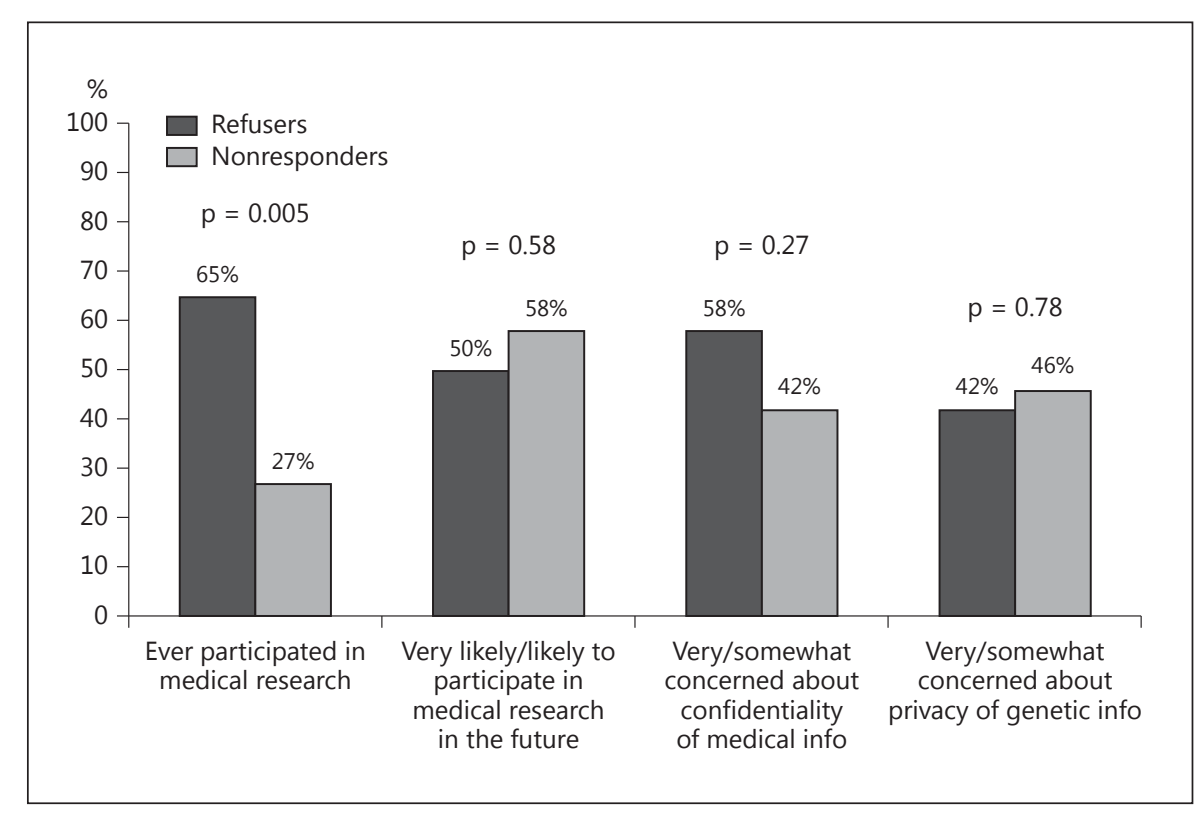

large profit from it, rather than using it purely for research', and one person said that the materials led him to believe that he would incur medical costs by participating.

While both groups answered similarly on questions about confidentiality of medical information and privacy of genetic information, refusers were more likely to cite this as the reason for their nonparticipation. In particular, they noted concerns about sharing information with researchers outside the Mayo Clinic, concerns about electronic data storage and concerns about information getting into the wrong hands, e.g. people that make insurance decisions.

\section{Discussion}

Our study sought to identify characteristics of biobank participants, refusers and nonresponders so that we could begin to understand potential nonresponse bias and decision factors for the latter 2 groups. We found that there are differences not only between participants and nonparticipants, but between refusers and nonresponders. Women and older patients were more likely to respond to the biobank recruitment packet, whether to participate in the biobank or to notify the staff that they were refusing, than to ignore the biobank invitation altogether. We had hoped that the simplified consent form designed for this study would increase participation and appeal to young patients in particular, but our results did not provide any evidence that it resulted in recruitment gains. If biobanks strive to ensure adequate representation of men,

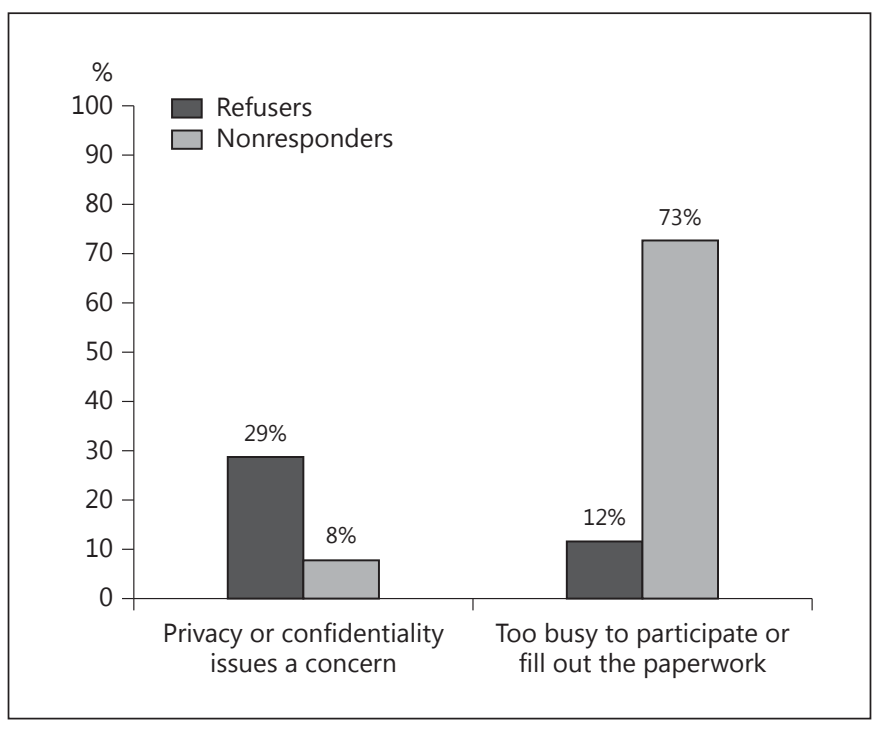

Fig. 4. Reasons for nonparticipation, refusers and nonresponders. Refusers and nonresponders were asked to report the reason for their participation decision. Responses were coded to these themes and frequencies were compared between groups.

younger patients and minority patients, oversampling or strategies other than mere modification of the consent process may be necessary.

Generally we saw that individuals who actively refused to participate in the biobank were informed and engaged patients. They were just as likely as participants and nonresponders to have heard about the biobank in the past, 
and they actively sought information about the biobank. One in 4 refusers said they looked for information to help them make a decision, and two-thirds said they read all or most of the consent form - nearly as many as in the participant group. Although our interview numbers were too small to detect a significant difference (13 refusers who received the original form and 13 that received the simplified version), we did find that a higher share of refusers who received the simplified form read all or most of it than refusers who received the original form (84 vs. $53 \%, p=0.20$ ), suggesting that those refusers may have had more information about the biobank.

If these patients make nonparticipation decisions after less complete review of the mailed materials and if further review would have led them to agree to participate based on better comprehension, the biobank staff may need to consider the delivery of key messages up front or in a manner that is more easily and immediately understood. Likewise, assessment of perceived burden deserves more attention. Both the original and simplified consent forms were accompanied by several other materials, including the health questionnaire. Future research should better assess which participation requirements or recruitment materials add to the perception of burden, thus, helping the biobank staff determine an acceptable level of burden in the nonresponder group. This information would be beneficial to future recruitment efforts.

The level to which refusers and nonresponders were engaged in information-seeking behaviors related to the biobank also helps explain findings on confidentiality concerns. Refusers and nonresponders were equally concerned about the confidentiality of their medical information and the privacy of their genetic information, but only refusers, who were far more likely to read the consent materials, cited that as a reason for nonparticipation. Nonresponders, on the other hand, may have been too busy to read the materials enough to understand the potential risks around data disclosure. While it is always important for patients to fully read and comprehend the informed consent materials before they decide to participate in research, failing to understand and adequately consent to biobank participation may raise unique concerns given the unknowns regarding future use of samples. Poor comprehension could also lead to poorly informed nonparticipation decisions. Issues like profit-making, costs and risks to data privacy were addressed in our consent document, but the interviews with nonparticipants revealed that some respondents did not accurately recall that information. Research by Han et al. [12] shows that participants perform poorly on key questions about information in the consent

Biobank Nonresponse, Refusal and Participation document (regardless of whether the patient received the original or the simplified form), but it is possible that refusers are better informed. Future assessment of consent comprehension among nonparticipants could add to understanding on this issue, including whether there are key issues about biobanking that are most influential specifically in refuser and nonresponder decision-making.

Our findings are consistent with other studies that identified concern about confidentiality and privacy of information, concerns about data sharing and trust in the institution as issues, but we add to the literature by identifying reasons for nonresponders and refusers separately and in a real-world recruitment situation. An important strength of this study was that we were able to observe actual participation decisions. Our results showing refusers were more likely than nonresponders to participate in research in the past but equally likely to say they would participate in the future confirms that hypothetical research questions may give biased results.

While our study has a number of strengths, potential limitations warrant mention and consideration. First, Mayo Clinic patients may have built a trust with the Mayo Clinic that may or may not translate to biobanks constructed in other contexts and/or for other purposes. These issues may be especially important for biobanks where participation involves a blood draw, a health questionnaire and access to medical record data into an unknown future. Second, due to staffing limitations, not all patients received a follow-up phone call if they did not respond to the recruitment packet within 14 days, as was the protocol. However, we explored the influence of this phone call in multivariate analysis and found that this did not affect our findings. Third, we did not follow-up with a representative sample of refusers and nonresponders. Rather, these were individuals we were able to contact through phone follow-up and who agreed to be interviewed. As such, they are not complete abstainers from research by the very nature of this inclusion criterion. Furthermore, we limited the telephone interviews in length to reduce barriers to recruitment in the interview, but more in-depth interviews would likely have yielded richer data to inform our results and in-person interviews would have allowed for greater probing on issues related to materials and remove concerns about interviewee recall. Nonetheless, we believe that this limitation is not unique to this particular investigation. Finally, our research is restricted to requests to participate in a single biobank at one point in time. Findings may not be fully generalizable, but they may apply to other biobanks that recruit patients by mail coordinated with a scheduled clinical visit. 
In conclusion, significant associations between patient characteristics and nonresponse, as well as systematic differences between refusers and nonresponders, could introduce bias in biobank research. Oversampling or changes in biobank recruitment procedures, including heightened attention to privacy protection, may be necessary to increase participation among less-represented groups if the biobank is to be a large, diverse resource for genetic research. Future research with a wide range of patient groups, including young men and people from a variety of racial or ethnic groups, should be undertaken to supplement the small but burgeoning literature on reasons for biobank participation, refusal and nonresponse.

\section{Acknowledgements}

The authors would like to thank the staff of the Mayo Clinic Biobank, especially Jody Morrisette and Kari Anderson as well as the staff of the Mayo Clinic Survey Research Center and the Biomedical Ethics Research Unit, especially Marguerite Robinson. This research was presented at the Academy Health Annual Research Meeting, June 24-26, 2012, Orlando, Fla. The project described was supported by Grant Number U01 HG04599 from the National Human Genome Research Institute and by CTSA Grant Number UL1 TR000135 from the National Center for Advancing Translational Sciences (NCATS), a component of the National Institutes of Health (NIH). This project was additionally supported by the Mayo Clinic Center for Individualized Medicine.

\section{References}

1 DiMartino L, Allen KD, Kasarskis E, Lindquist JH, Coffman CJ, Oddone EZ; National Registry of Veterans with ALS: Characteristics associated with participation in DNA banking: The National Registry of Veterans with ALS. Contemp Clin Trials 2007;28:572-582.

-2 Sanner JE, Frazier L: Factors that influence characteristics of genetic biobanks. J Nurs Scholorship 2007;39:25-29.

-3 Meisel SF, Shankar A, Kivimaki M, Wardle J: Consent to DNA collection in epidemiological studies: findings from the Whitehall II cohort and the English Longitudinal Study of Ageing. Genet Med 2012;14:201-206.

4 Kettis-Lindblad A, Ring L, Viberth E, Hansson MG: Genetic research and donation of tissue samples to biobanks. What do potential sample donors in the Swedish general public think? Eur J Public Health 2006;16:433-440.

5 Pentz RD, Billot L, Wendler D: Research on stored biological samples: views of African American and White American cancer patients. Am J Med Genet A 2006;140:733-739.

6 Trinidad SB, Fullerton SM, Bares JM, Jarvik GP, Larson EB, Burke W: Genomic research and wide data sharing: views of prospective participants. Genet Med 2010;12:486-495.

-7 Streicher SA, Sanderson SC, Jabs EW, Diefenbach M, Smirnoff M, Peter I, Horowitz CR, Brenner B, Richardson LD: Reasons for participating and genetic information needs among racially and ethnically diverse biobank participants: a focus group study. J Community Genet 2011;2:153-163.

8 Goddard KAB, Smith KS, Chen C, McMullen C, Johnson C: Biobank Recruitment: Motivations for Nonparticipation. Biopreserv Biobank 2009;7:119-121.
-9 McCarty CA, Chapman-Stone D, Derfus T, Giampietro PF, Fost N; Marshfield Clinic PCAG: Community consultation and communication for a population-based DNA biobank: the Marshfield clinic personalized medicine research project. Am J Med Genet A 2008;146A:3026-3033.

10 Melas PA, Sjöholm LK, Forsner T, Edhborg M, Juth N, Forsell Y, Lavebratt C: Examining the public refusal to consent to DNA biobanking: empirical data from a Swedish population-based study. J Med Ethics 2010;36: 93-98.

11 Lemke AA, Wolf WA, Hebert-Beirne J, Smith ME: Public and biobank participant attitudes toward genetic research participation and data sharing. Public Health Genomics 2010; 13:368-377.

12 Han LC, Ridgeway JL, Ziegenfuss JY, Robinson ME, Lackore KA, Olson JE, Koenig BA: Is more always better? The impact of simplified informed consent on response rates and comprehension in a clinic-based biobank (paper session 410). Annu Meet Am Soc Bioethics and Humanities, Washington, D.C., October 2012.

13 Agency for Healthcare Research and Quality (AHRQ): Sample Informed Consent Form. U.S. Department of Health and Human Services. http://www.ahrq.gov/fund/informedconsent/icform1.htm (accessed November 10, 2010)

14 Beskow LM, Burke W, Merz JF, Barr PA, Terry S, Penchaszadeh VB, Gostin LO, Gwinn M, Khoury MJ: Informed consent for population-based research involving genetics. JAMA 2001;286:2315-2321.
15 Beskow LM, Clayton EW, Eisenberg L, Henriksen-Hellyer J, McCarty C, McGuire AL, Smith ME, Somkin CP, Waudby C, Wolf W: Model Consent Language. The Electronic Medical Records and Genomics (eMERGE) Network Consent \& Community Consultation Workgroup Informed Consent Task Force, December 15, 2009. http://www.genome.gov/27526660.

16 Beskow LM, Friedman JlY, Hardy NC, Lin L, Weinfurt KP: Developing a simplified consent form for biobanking. PLoS One 2010; 5:e13302.

17 Jefford M, Moore R: Improvement of informed consent and the quality of consent documents. Lancet Oncol 2008;9:485-493.

18 National Cancer Institute: Simplification of Informed Consent Documents http://www. cancer.gov/clinicaltrials/understanding/simplification-of-informed-consent-docs/page2 (accessed November 10, 2010).

19 Office of Biorepositories and Biospecimen Research, National Cancer Institute, National Institutes of Health, U.S. Department of Health and Human Services: Custodianship and Ownership Issues In Biospecimen Research Symposium - Workshop. Rockville, October 4-5, 2007

20 US Department of Health and Human Services: Plain language, a promising strategy for clearing communicating health information and improving health literacy. 2005. http:// www.health.gov/communication/literacy/ plainlanguage/IssueBrief.pdf.

21 Teddlie C, Tashakkori A: Foundations of mixed methods research: integrating quantitative and qualitative approaches in the social and behavioral sciences. Thousand Oaks, SAGE, 2009. 